\title{
MAGNETISMO Y CAMBIOS AMBIENTALES EN REGISTROS TERRESTRES: EL LAGO DE MARBORÉ, PARQUE NACIONAL DE ORDESA Y MONTE PERDIDO (HUESCA)
}

\author{
B. OLIVA-URCIA ${ }^{1 *}$, A. MORENO ${ }^{1}$, \\ B. VALERO-GARCÉS ${ }^{1}$, P. MATA ${ }^{2}$, \\ Grupo HORDA $1,2,3,4$
}

\begin{abstract}
Grupo HORDA: Aida Adsuar, Josu Aranbarri, Fernando Barreiro, Miguel Bartolomé, Beatriz Bueno, Eduardo García-Prieto, Begoña García, Graciela Gil, Penélope González, Juan Cruz Larrasoaña, Josep M. Parés, Ana Pérez, Mayte Rico, Ariadna Salabarnada, Ángel Salazar, Miguel Sevilla-Callejo, Pol Tarrats.

${ }^{1}$ Instituto Pirenaico de Ecología, CSIC, Campus Aula Dei, Av. Montañana 1005, 50059 Zaragoza. ${ }^{2}$ Instituto Geológico y Minero de España (IGME), C/ La Calera 1, Tres Cantos, 28760 Madrid. ${ }^{3}$ Universidad de Barcelona, Fac. Biologia, Avinguda Diagonal 643, 08028 Barcelona. ${ }^{4}$ Centro Nacional de Investigación sobre la Evolución Humana, Paseo Sierra de Atapuerca, s/n, 09002 Burgos.
\end{abstract}

RESUMEN. El estudio de las propiedades magnéticas y sus variaciones en el tiempo en sondeos lacustres permiten deducir cambios paleoambientales de los últimos milenios. Este estudio presenta una aproximación multiproxy integrando indicadores geoquímicos y sedimentológicos con propiedades magnéticas analizadas en un sondeo largo $(\sim 6 \mathrm{~m})$ del Lago de Marboré a 2500 m.s.n.m. en el Pirineo Centro-Occidental que cubre los últimos $\sim 12.7 \mathrm{ka}$ cal BP. Facies, composición geoquímica y propiedades magnéticas identifican claramente ambientes sedimentarios con gran influencia glaciar en el periodo 12.7-11.7 ka cal BP, incluyendo el Younger Dryas (YD). Estos periodos más fríos (YD) están caracterizados por menor contenido en minerales ferromagnéticos s.l. El comienzo del Holoceno está marcado por un ligero aumento de la bioproductividad en el lago. Durante el periodo 9.2-4.1 ka cal BP el aumento de la bioproductividad, la mayor presencia de carbonatos detríticos, y una disminución generalizada en la concentración y variedad de minerales ferromagnéticos s.l. son coherentes con un periodo de clima más húmedo. La transición ca. 5-4.5 ka cal BP es el cambio más significativo de todo el Holoceno caracterizada por un aumento en la concentración de los minerales magnéticos, un descenso en TOC y de carbonatos detríticos que se interpretan como una respuesta al incremento de la aridez.

\section{Magnetism and environmental changes from terrestrial core of the lake Marboré, Parque Nacional de Ordesa y Monte Perdido (Huesca)}

ABSTRACT. The study of the magnetic properties and their variations with time in lacustrine sediment cores allow deducing paleoenvironmental changes in the last 
millennia. This study presents a multiproxy approach that integrates geochemical, sedimentological and magnetic properties records from a long core ( $6 \mathrm{~m})$ of the Marboré Lake at 2500 m. a.s.l. in the West-central Pyrenees. The core spans over the last $12.7 \mathrm{ky} \mathrm{cal} \mathrm{BP.} \mathrm{Facies,} \mathrm{geochemical} \mathrm{composition} \mathrm{and} \mathrm{magnetic} \mathrm{properties}$ clearly identify sedimentological environments with a great glaciar influence from 12.7-11.7 ky cal BP, including the Youger Dryas. These colder periods (YD) are characterized by a lower content in ferromagnetic minerals s.l. The beginning of the Holocene is marked by a slightly increase in the lake bioproductivity. During 9.2$4.1 \mathrm{ky} \mathrm{cal} \mathrm{BP}$ the increase in bioproductivity, higher content of detrital carbonates and a general decrease in the concentration and variety of the ferromagnetic s.l. minerals are coherent with a more humid period. The transition ca. 5-4.5 ky cal BP is the most significant change during the whole Holocene and it is characterized by an increase in the ferromagnetic minerals s.l., a decrease in TOC and detrital carbonates, which are interpreted as a response to an increase of aridity.

Palabras clave: magnetismo ambiental, sedimentología lacustre, geoquímica inorgánica, Pirineos, Holoceno.

Key words: environmental magnetism, lacustrine sedimentology, inorganic geochemistry, Pyrenees, Holocene.

Enviado el 15 de noviembre de 2012

Aceptado el 14 de enero de 2013

* Correspondencia: Instituto Pirenaico de Ecología, CSIC, Av. Montañana 1005, 50059 Zaragoza. E-mail: boliva@ipe.csic.es

\section{Introducción}

El Holoceno (últimos 11700 años) representa el interglacial actual, un periodo cálido y relativamente húmedo que se ha considerado estable en comparación con el estadio glacial anterior (de duración entre hace 120000 y 11700 años). Aunque el rango de variación de temperatura durante el Holoceno ha sido menor que en periodos glaciares (Johnsen et al., 1992), las reconstrucciones en ambientes continentales a partir de archivos lacustres han permitido describir grandes variaciones en la humedad y el balance hidrológico, a escala global (Cohen, 2003) y regional (Morellón et al., 2009), tanto a escala de milenios (Moreno et al., 2011) como de pocos cientos de años (Pérez Sanz et al., 2011). Así, en la Península Ibérica se han observado condiciones más húmedas durante el Holoceno Temprano en contraste con los milenios posteriores cuando dominaron condiciones de relativa aridez (Morellón et al., 2009), en clara relación con las variaciones en la insolación estacional controlada por los cambios en los parámetros orbitales. A pesar de que esta transición hacia una aridificación relativa del Holoceno Medio (aproximadamente entre 7000 y 5000 años antes del presente) está bien establecida, las características de este intervalo (duración, inicio, intensidad...) están por caracterizar. Profundizar en el conocimiento de los cambios climáticos de los últimos milenios requiere (1) seleccionar registros de 
ambientes especialmente sensibles al cambio climático, como pueden ser los ambientes de montaña (Plà y Catalán, 2005) y (2) analizar múltiples indicadores que, combinados, permitan extraer la señal hidrológica que, en muchos casos, es difícil de detectar por la creciente actividad humana y la consiguiente modificación del paisaje (Moreno et al., 2011). Entre los diferentes indicadores que se usan para explorar la variabilidad climática del pasado, las propiedades magnéticas de los sedimentos aparecen como claros marcadores de cambios ambientales y/o antropogénicos (Lanci et al., 1995; Kodama et al., 1997; Liu et al., 1999; Evans y Heller, 2003).

Desde el primer estudio de las propiedades magnéticas de sedimentos lacustres cuaternarios varvados de Suecia realizado por Ising en 1927 (Maher y Thompson, 1999), el estudio de las propiedades magnéticas en lagos se ha aplicado a sedimentos con el fin de entender cómo los procesos de transporte, depósito, diagénesis, disolución, erosión, escorrentía, cantidad de lluvia, intensidad del viento, etc., están en relación con cambios ambientales, paleoclimáticos y/o antropogénicos. La disciplina de magnetismo ambiental ha dado buenos resultados como un indicador paleoclimático más en estudios mutidisciplinares que combinan desde estudios geoquímicos (fluorescencia de rayos $\mathrm{X}$, análisis cuantitativos de elementos geoquímicos -ICP masas-, análisis isotópicos) a biológicos (distribución de las masas arbóreas a través del polen, de organismos acuáticos como diatomeas y quironómidos) (Smol et al., 2001). La combinación de los diferentes marcadores ambientales es posible realizarla a través de un buen control temporal de las series estudiadas mediante dataciones $\mathrm{Pb} / \mathrm{Cs}$ (hasta $\sim 150$ años) $\mathrm{o}^{14} \mathrm{C}$ (hasta $\sim 50 \mathrm{ka}$ ).

Los lagos suponen un repositorio natural de información ambiental ya que los materiales que se depositan en ellos provienen de la atmósfera, de la escorrentía o de los procesos diagenéticos y biogénicos que tienen lugar en el mismo (dependiendo del tipo de cuenca lacustre, características limnológicas del lago, etc). Asimismo, los procesos diagenéticos debidos a las características físico-químicas del fondo del lago, pueden disolver y/o precipitar minerales (entre ellos los ferromagnéticos) ya que son muy sensibles a los cambios en las condiciones redox. En tal caso, la información paleoambiental proporcionada por los sedimentos se referirá a la evolución hidroquímica del lago principalmente. El estudio magnético de sedimentos lacustres se ha realizado de forma extensiva desde 1975 (Maher y Thompson, 1999) aunque en la Península Ibérica son escasos los estudios donde se analiza en profundidad este marcador magnético (Larrasoaña et al., 2010; Borruel Abadía, 2012).

En este trabajo presentamos los resultados de los análisis magnéticos llevados a cabo en un sondeo largo ( $\sim \mathrm{m})$ realizado en el Lago de Marboré (Parque Nacional de Ordesa y Monte Perdido, Pirineo Central) en combinación con análisis geoquímicos para establecer la evolución ambiental del lago en los últimos $\sim 12.7$ ka.

\section{Localización geológica y morfología glaciar}

El Lago de Marboré se sitúa en la zona central de las Sierras Interiores pirenaicas (Fig. 1). El relieve del Pirineo viene dado en primera instancia por la tectónica alpina que 


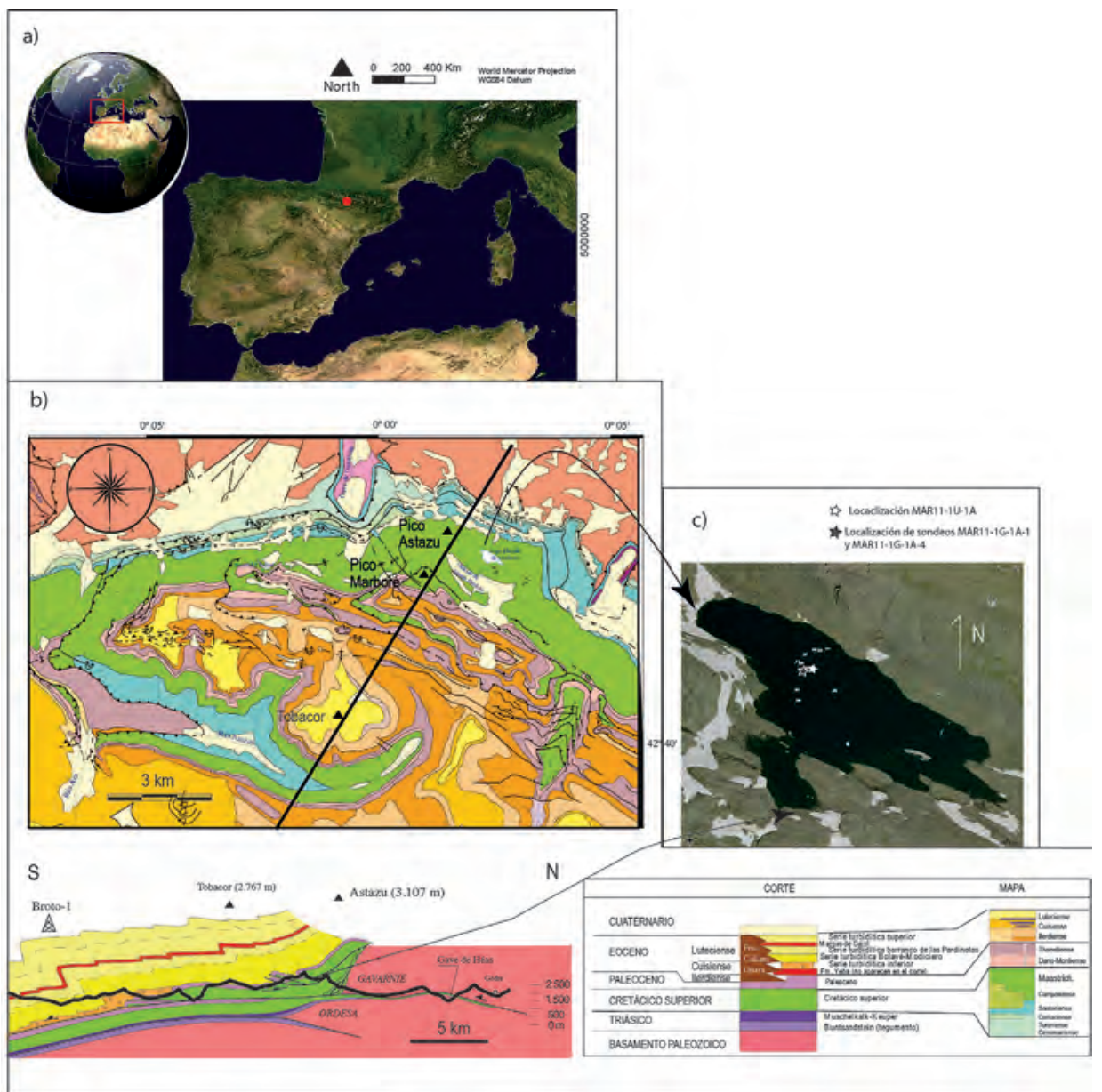

Figura 1. a) Localización del Lago de Marboré en Iberia. b) Situación y cortes geológicos (Ríos et al., 1987; Oliva Urcia, 2004). c) Ortoimagen donde aparece la situación de los sondeos realizados en la campaña de 2011.

conforma la estructura que se desarrolla debida al acortamiento de la cadena pirenaica por el acercamiento de la placa africana y la europea (entre el final del Cretácico superior y el Mioceno, Muñoz, 1992). Pero es durante los últimas decenas de miles de años cuando el modelado glaciar y kárstico configuran el relieve tal y como lo vemos hoy en día. Las Sierras Interiores constituyen la alineación montañosa de materiales Mesozoico-Terciarios carbonatados que bordean por el sur a la Zona Axial Pirenaica y que están limitadas al sur por la cuenca turbidítica de Jaca-Pamplona. La estructura de las Sierras Interiores viene definida por un cinturón de pliegues y cabalgamientos de rumbo WNW-ESE con buzamientos variables. Este primer sistema de cabalgamientos de Larra-Monte Perdido afecta a la serie del Cretácico Superior y a la serie calcárea y turbidítica Terciaria produciendo el acortamiento de la cobertera entre el Bartoniense y el Priaboniense (Montes, 
1992; Soler y Puigdefàbregas, 1970; Teixell, 1992). El avance de la deformación produce el apilamiento de cabalgamientos de basamento que deforman a los anteriores en secuencia de bloque inferior (Parish, 1984; Labaume et al., 1985; Cámara y Klimowitz, 1985). Así, entre el Eoceno y el Oligoceno inferior se emplaza el cabalgamiento de basamento de Gavarnie (Labaume et al., 1985; Teixell, 1996) y entre el Oligoceno-Mioceno inferior se emplaza el cabalgamiento de basamento de Guarga (Pocoví et al., 1990; Millán et al., 1995; Arenas et al., 2001).

En concreto, el Lago de Marboré se sitúa en el eje de un sinclinal dentro de la Fm. Areniscas de Marboré (rocas de plataforma carbonatada del Cretácico Superior -Campaniense-Maastrichtiense) a 2500 m de altitud, en el Balcón de Pineta (circo de Marboré), limitado al norte por el Pico de Tucarroya $(\sim 2800 \mathrm{~m})$, al W por los Picos Astazus ( 3000 m) y al S por el Pico de Monte Perdido (3355 m). El Lago de Marboré desagua hacia el E, hacia el valle de Pineta (río Cinca). La erosión glaciar del circo de Marboré y la evolución kárstica del mismo ponen de manifiesto el relieve estructural y controlan la evolución del lago desde la última deglaciación. El lago presenta unas dimensiones de $\sim 500 \mathrm{~m}$ en la dirección de las Sierras y $200 \mathrm{~m}$ en la dirección perpendicular. Existe una represa del año 1938 (Iberduero) que está decomisionada en al actualidad (Fig. 1).

El máximo glaciar global (23-19 ka) corresponde en la Península Ibérica con una pulsación fría pero no con la máxima extensión de los glaciares de montaña (GarcíaRuiz et al., 2010). Esta máxima extensión de los glaciares ocurrió en los valles pirenaicos occidentales (Aragón, Gállego) y centrales (Cinca) entorno a los 65000 años, como apuntan los últimos estudios (García-Ruiz et al., 2003; González-Sampériz et al., 2006; Lewis et al., 2009). En el valle de Pineta donde se localiza el Lago de Marboré se ha datado el techo del relleno de un lago de obturación glacial, situado a menor altitud, en el valle de La Larri en 13245 +/- 120 años cal. BP (Salazar et al., 2011). El valle de La Larri es un valle glaciar colgado situado unos $4 \mathrm{~km}$ al NE del Lago de Marboré. Esta datación sugiere que el glaciar de Pineta se había retirado prácticamente hasta el umbral del balcón de Pineta hace unos 13 ka (Salabarnada, 2011), permitiendo por tanto la formación del Lago de Marboré.

Los depósitos glaciares y morrénicos del circo de Marboré han sido objeto de estudio desde los años 80, junto con el glaciar de Monte Perdido, uno de los últimos relictos glaciares del Pirineo (Nicolás-Martínez 1981; Martínez de Pisón y Arenillas 1988; García-Ruiz et al. 2001; Chueca et al., 2002). En el circo de Pineta se distinguen dos tipos de morrenas: i) morrenas grises con grandes cantos (6-8 $\mathrm{m}$ de diámetro) con una edad asignada al Holoceno temprano y ii) morrenas de color marrón con cantos pequeños del Maastrichtiense y del Paleoceno más recientes que las anteriores (García-Ruiz et al., 2001). Ambos tipos aparecen en la parte sur del circo, mientras que no se ha encontrado ningún resto morrénico en la parte norte, donde se sitúa el Lago de Marboré. Documentos históricos y fotografías sitúan en el extremo NW del ibón de Marboré un pequeño glaciar o nevero permanente que drenaba directamente al lago y que ha desaparecido en las últimas décadas. 


\section{Metodología}

\subsection{Obtención de sondeos, muestreo y análisis sedimentológicos y geoquímicos}

En Agosto de 2011 el equipo del proyecto HORDA obtuvo 3 sondeos largos ( $6 \mathrm{~m}$ cada uno) mediante una plataforma Uwitec y 16 sondeos cortos $(<1 \mathrm{~m})$ por gravedad en 5 puntos de muestreo diferentes del Lago de Marboré. En este trabajo se presentan los resultados de los análisis de las propiedades magnéticas y geoquímicas de la secuencia sedimentaria recuperada, formada por la correlación de los sondeos MAR111G-1A-1, y MAR11-1U-1A. Ambos sondeos se abrieron, fotografiaron y muestrearon en el laboratorio del IPE-CSIC en Zaragoza en Septiembre de 2011. Los análisis de Carbono orgánico total (TOC) y Carbono Inorgánico (TIC) se han determinado cada $2 \mathrm{~cm}$ por absorción de infrarrojos del $\mathrm{CO}_{2}$ total liberado por combustión en un analizador LECO SC144 DR.

El análisis mediante la fluorescencia de rayos X (FRX) por medio de un Core Scanner modelo AVAATECH XRF II (Universidad de Barcelona) permite obtener en continuo información cualitativa sobre la composición geoquímica del sedimento (elementos químicos) del sondeo. El espaciado de muestreo seleccionado fue de $5 \mathrm{~mm}$ y los análisis se realizaron a $10 \mathrm{kV}$, con 1300 micro Amperios con un tiempo de $10 \mathrm{~s}$ y sin filtro para $\mathrm{Al}$, Si, K, Ca, Ti, V, Cr, Mn, Fe, y a 30 kV la radiación de 2000 micro Amperios con un tiempo de 30 s y con filtro de Pd (paladio) para Zn, Ga, As, Br, Rb, Sr, Y, Zr, Nb y Pb. Los resultados se representan en cuentas por segundo a lo largo del sondeo y se han calculado las relaciones entre parejas de elementos (Tabla 1).

Tabla 1. Relación $R^{2}$ entre elementos. En fondo azul aquellas relaciones mayores de 0.4.

\begin{tabular}{|c|c|c|c|c|c|c|c|c|c|c|c|}
\hline & $\mathbf{A l}$ & $\mathbf{S i}$ & $\mathrm{Ti}$ & $\mathbf{V}$ & $\mathbf{P b}$ & $\mathbf{R b}$ & $\mathbf{Z r}$ & $\mathbf{F e}$ & $\mathbf{C a}$ & $\mathbf{M n}$ & $\mathbf{S r}$ \\
\hline $\mathrm{K}$ & 0.87251 & 0.68424 & 0.45499 & 0.31489 & 0.00038 & 0.64661 & 0.23286 & 0.24457 & 0.02197 & 0.10457 & 0.06177 \\
\hline $\mathrm{Al}$ & & 0.88103 & 0.49797 & 0.24327 & 0.00357 & 0.47631 & 0.11231 & 0.10898 & 0.0035 & 0.09726 & 0.08691 \\
\hline $\mathrm{Si}$ & & & 0.66447 & 0.18567 & 0.00316 & 0.27101 & 0.01833 & 0.05231 & $1.70 \mathrm{E}-05$ & 0.0773 & 0.06927 \\
\hline $\mathrm{Ti}$ & & & & 0.1852 & 0.00302 & 0.15042 & $2.50 \mathrm{E}-05$ & 0.07658 & 0.00425 & 0.04852 & 0.02252 \\
\hline $\mathrm{V}$ & & & & & 0.00011 & 0.24378 & 0.09453 & 0.12688 & 0.02219 & 0.03664 & 0.00451 \\
\hline $\mathrm{Pb}$ & & & & & & 0.02685 & 0.02343 & 0.12626 & 0.00735 & 0.02998 & 0.04629 \\
\hline $\mathrm{Rb}$ & & & & & & & 0.31379 & 0.10253 & 0.05074 & 0.16148 & 0.07041 \\
\hline $\mathrm{Zr}$ & & & & & & & & 0.40175 & 0.00012 & 0.00039 & 0.02016 \\
\hline $\mathrm{Fe}$ & & & & & & & & & 0.01588 & 0.10098 & 0.0102 \\
\hline $\mathrm{Ca}$ & & & & & & & & & & 0.09206 & 0.60641 \\
\hline $\mathrm{Mn}$ & & & & & & & & & & & 0.00055 \\
\hline
\end{tabular}




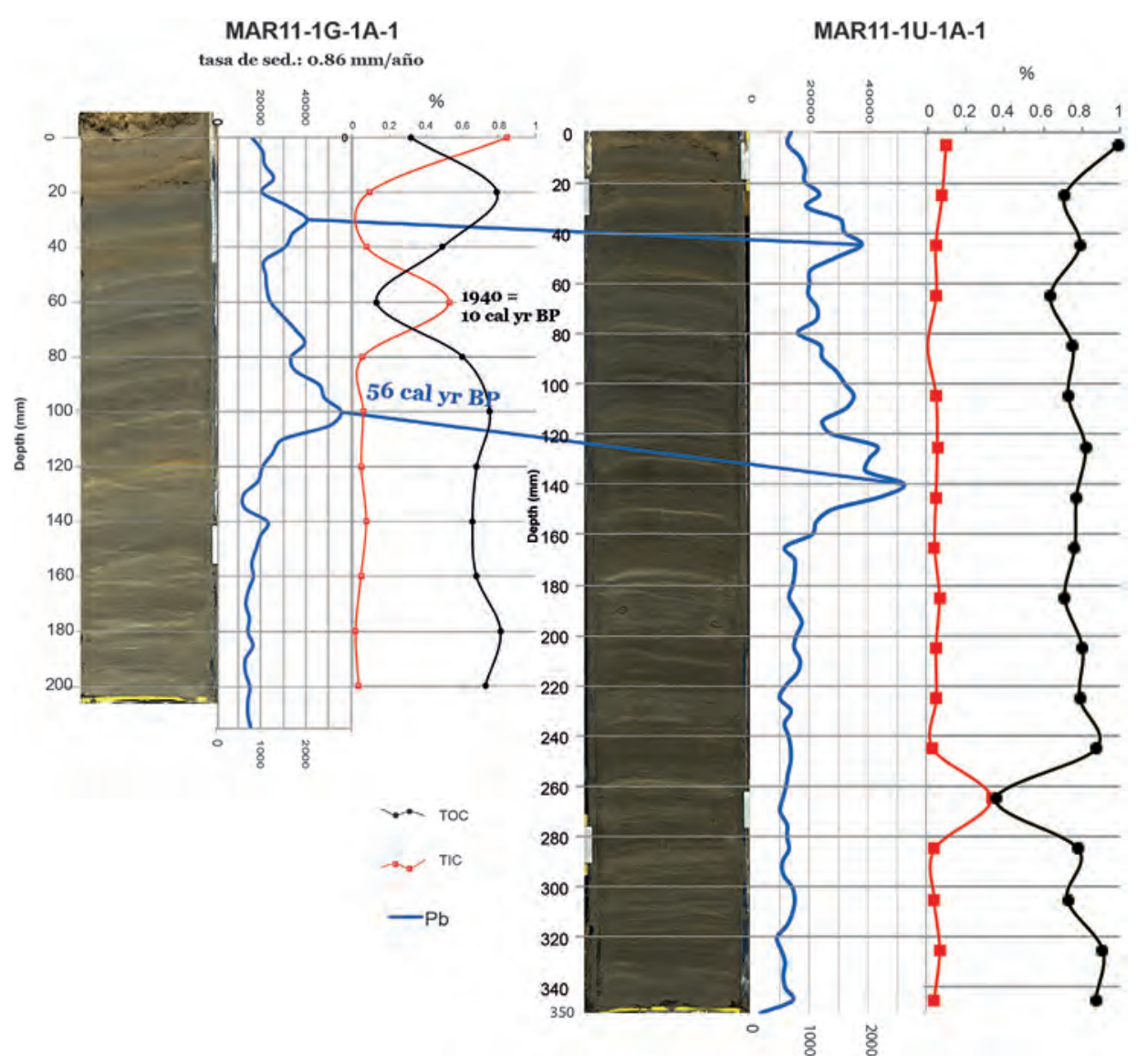

Figura 2. Correlación entre el sondeo corto (izda.) y el largo (dcha.) a partir de los picos de $\mathrm{Pb}$ de la fluorescencia de rayos $\mathrm{X}$.

El análisis de componentes del sedimento se ha realizado mediante el estudio de frotis por microscopía óptica, siguiendo la metodología propuesta por Myrbo et al. (2011) y la clasificación de Schnurrenberger et al. (2003).

\subsection{Correlación de sondeos y modelo de edad}

El modelo cronológico disponible de la secuencia sedimentaria del Lago de Marboré proviene de dataciones de ${ }^{14} \mathrm{C}$ y de la correlación con un sondeo corto donde se obtuvo la tasa de sedimentación para los sedimentos más recientes mediante técnicas radiométricas de datación absoluta con ${ }^{210} \mathrm{~Pb}$ y ${ }^{137} \mathrm{Cs}$. La correlación entre sondeos con los picos de TIC no es evidente, probablemente debido al escaso contenido en carbonato (aprox. 0.5\%). Sin embargo, la correlación entre el sondeo corto MAR11-1G-1A-1 y la sección superior del sondeo largo MAR11-1U-1A-1 utilizando la composición geoquí- 
mica obtenida por medio de la fluorescencia de rayos X (FRX), en concreto el elemento $\mathrm{Pb}$ (Fig. 2), es excelente. Esto nos permite asegurar que toda la sección de sedimento ha sido recuperada y que el techo de la secuencia del sondeo corto MAR11-1G-1A-1 corresponde a la interfase sedimento/agua (año 2011). La tasa de sedimentación media reciente ha sido calculada en $0.86 \mathrm{~mm} / \mathrm{año}$ en un sondeo corto (MAR11-1G-1A-4 equivalente al MAR11-1G-1A-1) mediante la datación con ${ }^{210} \mathrm{~Pb}$ y ${ }^{137} \mathrm{Cs}$.

Ante la imposibilidad de datar microcarbones o macrorestos vegetales dada su ausencia del sedimento, un total de 13 muestras de sedimento total del sondeo largo se han datado por medio de ${ }^{14} \mathrm{C}$ en el laboratorio Direct AMS de Seattle (WA) (Tabla 2). Las dataciones de ${ }^{14} \mathrm{C}$ se han calibrado utilizando el programa CALIB v.6, mediante la curva INTCAL09 (Reimer et al., 2009).

Tabla 2. Dataciones de AMS ${ }^{14} \mathrm{C}$ realizadas en 13 muestras de sedimento total de la secuencia del Lago de Marboré. Las muestras 1, 5 y 13 han sido descartadas por suponer una inversión en la secuencia sedimentaria.

\begin{tabular}{|c|c|c|c|c|c|c|c|}
\hline $\begin{array}{c}\mathbf{N}^{\mathbf{0}} \\
\text { orden }\end{array}$ & $\begin{array}{c}\text { Profundidad } \\
(\mathbf{c m})\end{array}$ & Lab ID & Código muestras & Material datado & $\begin{array}{c}\text { AMS }{ }^{14} \mathbf{C} \\
(\mathbf{a n ̃ o s})\end{array}$ & $\begin{array}{c}\text { Edad calibrada } \\
(\mathbf{2 \sigma}, \mathbf{9 5} \% \text { range })\end{array}$ & $\begin{array}{c}\text { Edad calibrada } \\
(\mathbf{m e d i a})\end{array}$ \\
\hline 1 & 15 & D-AMS 1217-203 & MAR11-1A-1U-1 8-10 cm & Sedimento total & $5771 \pm 46$ & $6453-6669$ & 6572 \\
\hline 2 & 325 & D-AMS 001189 & MAR11-1A-1U-1 cm 39-41 & Sedimento total & $2514 \pm 25$ & $2490-2643$ & 2588 \\
\hline 3 & 790 & D-AMS 1217-204 & MAR11-1A-1U-2 53-56 cm & Sedimento total & $3611 \pm 28$ & $3840-3983$ & 3920 \\
\hline 4 & 1675 & D-AMS 001190 & MAR11-1A-1U-2 cm 142-144 & Sedimento total & $4820 \pm 28$ & $5476-5541$ & 5521 \\
\hline 5 & 1990 & D-AMS 1217-205 & MAR11-1A-1U-3 27-30 cm & Sedimento total & $5853 \pm 30$ & $6615-6744$ & 6675 \\
\hline 6 & 2430 & D-AMS 1217-206 & MAR11-1A-1U-3 71-74 cm & Sedimento total & $5675 \pm 31$ & $6399-6542$ & 6455 \\
\hline 7 & 2735 & D-AMS 001191 & MAR11-1A-1U-3 cm 102-104 & Sedimento total & $6294 \pm 39$ & $7161-7308$ & 7220 \\
\hline 8 & 3230 & D-AMS 1217-207 & MAR11-1A-1U-4 17-21 cm & Sedimento total & $7464 \pm 55$ & $8183-8379$ & 8282 \\
\hline 9 & 3930 & D-AMS 001192 & MAR11-1A-1U-4 cm 88-90 & Sedimento total & $8447 \pm 39$ & $9420-9532$ & 9479 \\
\hline 10 & 4440 & D-AMS 1217-208 & MAR11-1A-1U-4 139-141 cm & Sedimento total & $9787 \pm 43$ & $11159-11262$ & 11214 \\
\hline 11 & 4770 & D-AMS 001193 & MAR11-1A-1U-5 cm 27-29 & Sedimento total & $10852 \pm 47$ & $12605-12887$ & 12722 \\
\hline 12 & 5030 & D-AMS 001194 & MAR11-1A-1U-5 cm 53-55 & Sedimento total & $11434 \pm 47$ & $13172-13417$ & 13303 \\
\hline 13 & 5635 & D-AMS 1217-209 & MAR11-1A-1U-5 $113-116 \mathrm{~cm}$ & Sedimento total & $11521 \pm 47$ & $13255-13484$ & 13365 \\
\hline
\end{tabular}

\subsection{Análisis de magnetismo ambiental}

El muestreo para las medidas de magnetismo ambiental se ha realizado mediante "u-channels", incrustando en el sedimento del sondeo previamente cortado longitudinalmente, una caja de longitud máxima de $1.5 \mathrm{~m}$ con una sección en forma de U de 2 $\mathrm{cm}$ de arista. Las medidas magnéticas se han realizado en el laboratorio de Arqueomagnetismo del Centro Nacional de Investigación, "Evolución Humana" (CENIEH, Burgos). Los análisis llevados a cabo combinan medidas de paleomagnetismo clásico y de magnetismo ambiental. Estas medidas se han realizado cada $2 \mathrm{~cm}$ en un magnetómetro 
criogénico $2 \mathrm{G}$ adaptado con un desmagnetizador por campos alternos para la desmagnetización progresiva hasta $100 \mathrm{mT}$ primero y para la aplicación de una remanencia anhisterética (ARM) con un DC de 50 nT y un AF de 100 mT después. Además se han obtenido medidas de saturación de la remanencia (IRM) por medio de un magnetizador de pulsos (2G Enterprises) a 150 mT, 300 mT después de la saturación en la dirección contraria de 1 T (Opdyke y Channel, 1996).

Asimismo se han representado las variaciones de la ARM y de las IRMs respecto a la profundidad, además del $S$-ratio $\left(\operatorname{IRM}_{300 \mathrm{mT}} / \mathrm{IRM}_{1 \mathrm{~T}}\right)$ y la HIRM $\left(\mathrm{IRM}_{1 \mathrm{~T}}-\mathrm{IRM}_{150 \mathrm{mT}}\right.$, $\mathrm{IRM}_{1 \mathrm{~T}}-\mathrm{IRM}_{300 \mathrm{mT}}$ ). La ARM representa la concentración de los minerales de coercitividad baja (magnetita o sulfuros de hierro que son ferrimagnéticos s.s.). La "susceptibilidad de la ARM" $\left(\chi_{\mathrm{ARM}}\right)$ se deriva normalizando la ARM con el campo DC aplicado y es un indicador de la concentración de granos ferrimagnéticos finos, monodominio estables (0.02$0.4 \mathrm{~m})$. La IRM $\mathrm{IO0mT}_{30}$ representa la concentración de los granos ferrimagnéticos mientras que la HIRM y la SIRM (IRM ${ }_{1000 \mathrm{mT}}$ ) reflejan la concentración de los minerales antiferromagnéticos (minerales de coercitividad alta: hematites, goethita) y del total de ferromagnéticos s.l. respectivamente (Oldfield, 1991; Hunt et al., 1995; Ortega et al., 2006; Wang et al., 2010). El S-ratio por el contrario pone de manifiesto la concentración de los minerales de mayor coercitividad respecto de los de baja coercitividad, cuanto más próximo a 1 es, los minerales de baja coercitividad son los que dominan la muestra (King y Channel, 1991; Verosub y Roberts, 1995; Peters y Dekkers, 2003). Además se utiliza como indicador de la mineralogía magnética, cuanto más próximo a 1 (valores de 0.9 ) es magnetita y cuanto más bajo (menos de $\sim 0.7$ ) mayor aporte de minerales de mayor coercitividad están presentes. Sin embargo, como recogen Liu et al. (2010), es necesario controlar esa variación a través de otro parámetro L-ratio (HIRM/0.5 x (SIRM + IRM ${ }_{-100 \mathrm{mT}}$ ), ya que sólo cuando el L-ratio experimenta pequeñas variaciones, se puede interpretar la HIRM y el $S$-ratio de forma convencional, como se ha explicado arriba. La $\mathrm{IRM}_{-100 \mathrm{mT}}$ representa la remanencia obtenida al saturar primero la muestra a $1 \mathrm{~T}$ y después aplicar un campo de $100 \mathrm{mT}$ en sentido contrario). En nuestro caso se ha aplicado un campo de $150 \mathrm{mT}$ en lugar de $100 \mathrm{mT}$.

Además se ha obtenido información sobre parámetros magnéticos (magnetización remanente -Mr-, magnetización de saturación -Ms-, coercitividad -Hc-, y coercitividad de la remanencia -Hcr-) cuyas relaciones indican el tamaño de grano magnético a partir del diagrama de Day (Day et al., 1977), gracias a los análisis realizados en el VSM (Vibrating Sample Magnetometer) del CENIEH (Burgos) y al tratamiento de los mismos por medio del paquete informático de L. Tauxe.

\section{Resultados}

\subsection{Facies Sedimentarias}

Los sedimentos recuperados del Lago de Marboré son muy homogéneos a lo largo de la secuencia sedimentaria. A grandes rasgos la mineralogía reconocida por el estudio microscópico está formada por cuarzo, filosilicatos, entre los que se reconocen clara- 
mente cristales de biotita, y carbonatos. La mayor parte del sondeo son facies laminadas, formadas por laminaciones milimétricas (en pocas ocasiones llegan a ser bandas centimétricas) de limos y lutitas de color marrón, ocre y gris compuestas esencialmente de cuarzo, minerales de la arcilla y otros silicatos con bajos porcentajes de materia orgánica y prácticamente sin carbonatos. Las laminaciones milimétricas observadas se deben principalmente a variaciones en el tamaño de grano y ligeras diferencias en la composición mineral. De esta manera, las láminas de color más claro son las de menor tamaño de grano (mayoritariamente dentro del rango del tamaño de arcilla, entre 2 y $5 \mu \mathrm{m}$ ) y con mayor contenido porcentual de cuarzo $(75 \%$, frente a $15 \%$ biotitas), mientras que las láminas marrones presentan un tamaño de grano mayor $(20-70 \mu \mathrm{m})$ y con mayor cantidad porcentual de biotitas (65\% cuarzo frente a $25 \%$ biotitas). En algunos casos, se observan láminas grisáceas que aparecen por debajo de las láminas blancas y que presentan un porcentaje similar a las blancas en cuanto a contenido mineral, pero el tamaño de grano es mayor (como en las láminas marrones, entre 20 y $60 \mu \mathrm{m}$ ).

\subsection{Geoquímica}

Los contenidos en carbono orgánico (0.1-1.1\%) y sobre todo inorgánico (0-0.4\%) son muy bajos a lo largo de toda la secuencia, lo que pone de manifiesto la baja cantidad de carbonato cálcico presente en las muestras analizadas y el escaso contenido orgánico típico en un lago de alta montaña (Fig. 3). Sin embargo, el contenido en TOC aumenta ligeramente (por encima de $0.7 \%$ ) en los dos metros superiores $(0-1835 \mathrm{~mm})$ y entre 2375 y $4590 \mathrm{~mm}$ de profundidad (por encima de $0.6 \%$ ).

Las variaciones de los elementos $\mathrm{K}, \mathrm{Al}, \mathrm{Si}, \mathrm{Ti}, \mathrm{V}, \mathrm{Pb}, \mathrm{Rb}, \mathrm{Zr}, \mathrm{Fe}, \mathrm{Ca}, \mathrm{Mn}$ y $\mathrm{Sr}$ son significativas en esta secuencia y se representan en profundidad en la Fig. 3. Las correlaciones encontradas (Tabla 1), nos permiten agrupar algunos elementos en función de su comportamiento: por un lado, los elementos $\mathrm{K}, \mathrm{Al}$, Si, Vy Ti están relacionados con el aporte de detríticos al lago (cuarzo, filosilicatos, minerales pesados) y varían de forma paralela a lo largo del sondeo, mostrando valores muy estables con claros mínimos en las capas con presencia de carbonatos. Por lo tanto, un mayor aporte detrítico viene marcado por el aumento de estos elementos. Otro grupo está formado por el $\mathrm{Ca}$ y el $\mathrm{Sr}$, asociados a la presencia de carbonatos en la secuencia, si bien no todos los intervalos carbonatados parecen tener la misma cantidad de Sr. Finalmente, dos elementos presentan comportamientos singulares: el $\mathrm{Mn}$ y el $\mathrm{Pb}$. El primero, es un elemento asociado a los cambios en las condiciones redox (Levy et al., 1992). En el caso de la secuencia del Lago de Marboré, el Mn parece ligado a las láminas carbonatadas probablemente porque su depósito supuso un cambio importante en las propiedades limnológicas. De hecho, dado que la roca donde se desarrolla el lago de Marboré es carbonatada (calcilutita, Fm. Areniscas de Marboré) es interesante observar la falta de Ca en el relleno sedimentario del lago (Fig. 3). El Ca sólo aparece por debajo de 2.4 m y simultáneamente a la presencia de picos de Mn (Fig. 3), lo que sugiere que la permanencia de carbonatos detríticos y de posibles óxidos de $\mathrm{Mn}$, se debe a procesos químicos, relacionados con cambios redox del lago en determinados momentos, posiblemente a la supersaturación en carbonatos del agua del lago. Las pequeñas desviaciones encontradas en los perfiles 


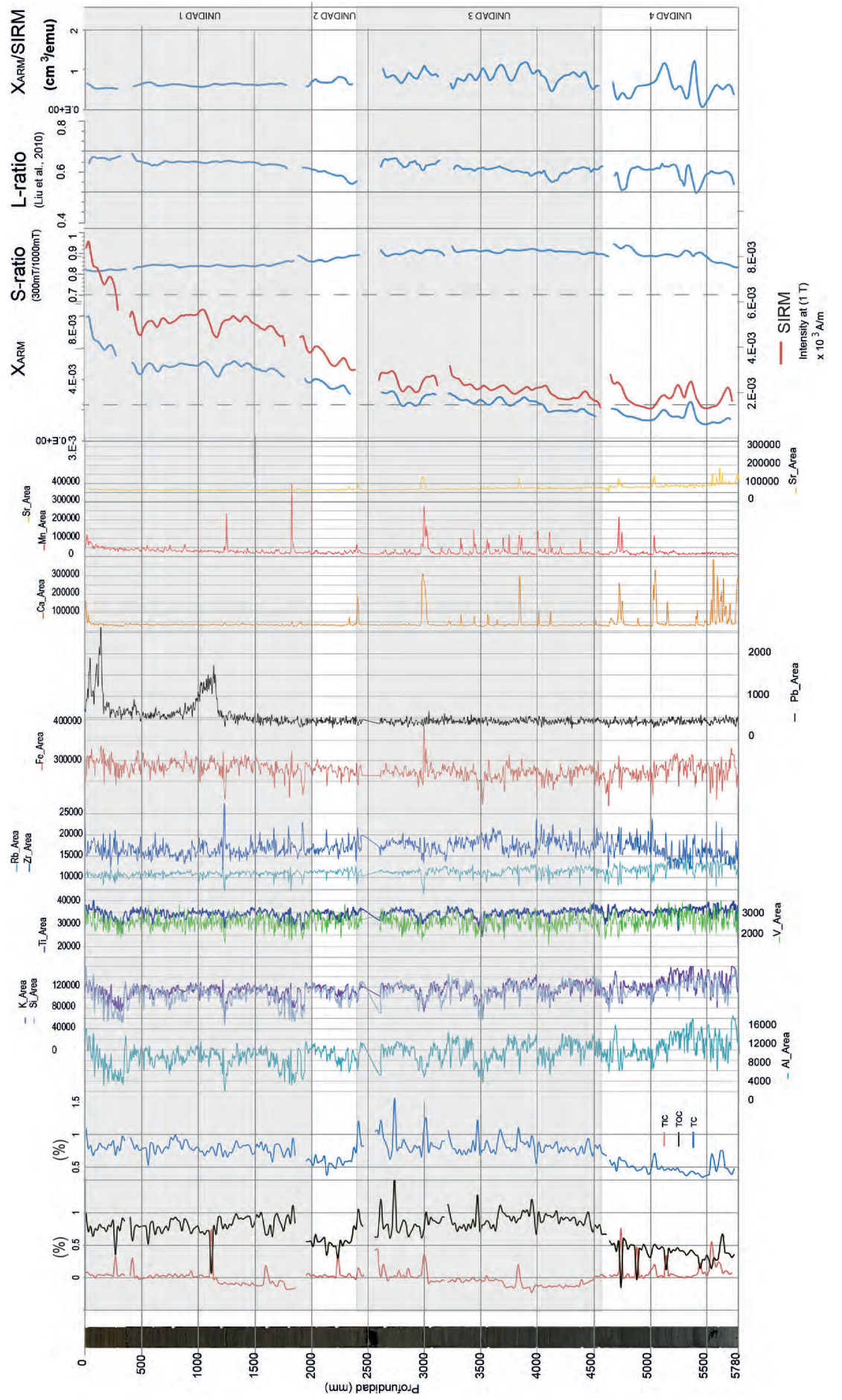


del Fe con respecto a los elementos ligados a la fracción detrítica, pueden deberse igualmente a procesos de cambios redox en el fondo del lago (ej. el pico relacionado con $\mathrm{Ca}$ y Mn del metro 3). Por último, el perfil del $\mathrm{Pb}$ es distinto al del resto de elementos analizados, presentando un valor bajo y estable a lo largo de toda la secuencia salvo en dos intervalos claramente diferenciados donde aumenta espectacularmente: los primeros 200 mm (200 años) y entre 1000 y 1250 mm (2000-2400 cal yr. BP).

\subsection{Magnetismo ambiental}

Se ha representado en la Fig. 3 (dcha.) una selección de las variaciones de las propiedades magnéticas más significativas, aunque se describen a continuación los valores de los parámetros analizados. Las variaciones de las propiedades magnéticas inducidas en los sedimentos indican un decrecimiento en la concentración de los minerales magnéticos con la profundidad. Esto se observa tanto en la curva de ARM, de $\chi_{\mathrm{ARM}} \mathrm{y}$ en las curvas de IRM (en la Fig. 3 se ha representado la $\chi_{\mathrm{ARM}}$ ). Este decrecimiento concomitante entre las diferentes curvas sugiere que es la concentración de minerales ferromagnéticos s.l. y no el tamaño de grano lo que varía con la profundidad (Ao et al., 2010).

Los valores de ARM varían entre $3 \times 10^{-4} \mathrm{emu} / \mathrm{cm}^{3}$ en la parte superior, mientras que por debajo de los $2800 \mathrm{~mm}$ los valores quedan por debajo de $1.2 \times 10^{-4} \mathrm{emu} / \mathrm{cm}^{3}$. Este aumento del valor de la ARM en la parte alta sugiere una mayor concentración de minerales ferromagnéticos de coercitividad baja, dado que hay mayor intensidad de la ARM aplicada en un campo alterno de $100 \mathrm{mT}$, en esa zona del sondeo.

Los valores de las diferentes IRMs son más distintos en la parte alta del sondeo, pero de nuevo, por debajo de los $2400 \mathrm{~mm}$ estos valores son más similares, lo que viene a corroborar los resultados del S-ratio y de HIRM. Los valores de las HIRM son también más elevados en la parte alta del sondeo, empezando en 2.5 ó $1.5 \times 10^{-3} \mathrm{emu} / \mathrm{cm}^{3}$, mientras que por debajo de la profundidad de $2400 \mathrm{~mm}$ las diferencias entre los valores de las HIRM representadas quedan igualadas, dando valores de 2.7 y 1.4 x $10^{-4} \mathrm{emu} / \mathrm{cm}^{3}$. Asimismo, las variaciones del $S$-ratio sugieren una concentración ligeramente mayor de los minerales de mayor coercitividad en la parte alta del sondeo. Esta observación del valor del S-ratio es posible realizarla dado que la variación del L-ratio es menor al $16 \%$ (Liu et al., 2010). Las variaciones de $\chi_{\mathrm{ARM}}$ /SIRM con la profundidad apoyan también que hay una mayor concentración de minerales en la parte alta del sondeo, ya que la relación ARM/SIRM (y por tanto la $\chi_{\mathrm{ARM}} / \mathrm{SIRM}$ ) aumenta según decrece la concentración, mostrando mayor interacción entre partículas finas (Sugiura, 1979). Las variaciones de la relación de $\chi_{\text {ARM }} /$ SIRM aumentan por debajo de la profundidad de $2400 \mathrm{~mm}$, sugiriendo junto con los datos anteriores una menor concentración de minerales ferromagnéticos s.l. por debajo de los $2400 \mathrm{~mm}$ de profundidad. Diferencias similares entre la parte superior del sondeo y la inferior se obtienen en los bi-plots donde se representan la ARM (y $\left.\chi_{\text {ARM }}\right)$, HIRM o $S$-ratio. Por encima de los $2400 \mathrm{~mm}$, hay una correlación positiva lineal entre la HIRM y la $\chi_{\mathrm{ARM}}$ con un $\mathrm{R}=0.97$. Mientras que la relación es negativa entre la HIRM y el $S$-ratio $(\mathrm{R}=0.97$, relación potencial). Por debajo de esa profundidad, la HIRM permanece prácticamente constante. Estos valores sugieren mayor 
Figura 4. Diagrama de Day et al., 1977 (modificado por Dunlop, 2002). Los resultados no siguen una línea que marcaría un determinado tamaño de grano.
Day diagram -MARBORÉ-

(Dunlop, 2002)

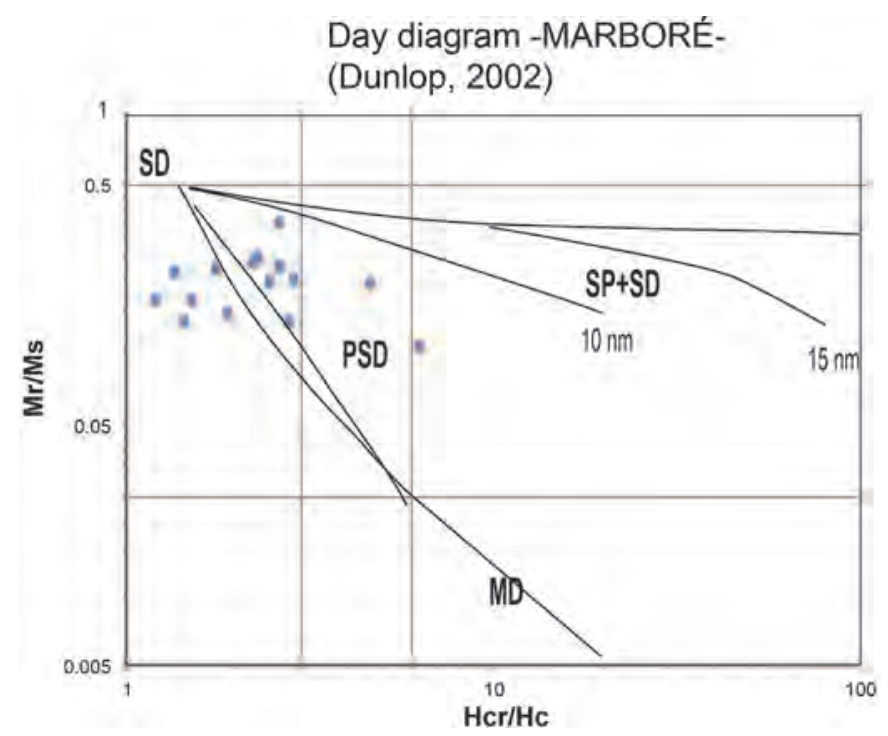

concentración de minerales ferromagnéticos de alta y baja coercitividad en la parte alta del sondeo, ya que a mayor valor de HIRM (mayor concentración de alta coercitividad) hay mayor valor de $\chi_{\text {ARM }}$ y menor valor de $S$-ratio (mayor concentración de minerales de baja coercitividad).

Los valores de la coercitividad de la remanencia (Hcr) obtenidos a partir de los ciclos de histéresis de muestras seleccionadas varía entre 46 y $10 \mathrm{mT}$ (valores de coercitividad de la remanencia bajos, propios de magnetita principalmente y de hematites ocasionalmente, Peters y Dekkers, 2003). Las relaciones entre Ms (magnetización de saturación) y Mr (magnetización de la remanencia) obtenidas también a partir de los ciclos de histéresis confirman que la variación de la concentración es lo que predomina a la lo largo del sondeo (Ao et al., 2010). Esta relación tiene una $\mathrm{R}=0.88$ en las muestras seleccionadas. A partir de los valores obtenidos en los ciclos de histéresis, se representa el diagrama de Day (Day et al., 1977) donde se puede observar que no hay una tendencia específica en cuanto a la variación del tamaño de grano (Fig. 4). Tampoco se observan ciclos de histéresis con las denominadas "cintura de avispa". Esta característica es propia de mezcla de granos ferromagnéticos s.l. de diferente coercitividad o tamaño de grano (Jackson, 1990; Channel y McCabe, 1994; Roberts et al., 1995; Tauxe et al., 1996).

\subsection{Unidades sedimentológicas}

Teniendo en cuenta las facies sedimentarias y la composición química y propiedades magnéticas de los sedimentos, la secuencia de Marboré se ha dividido en cuatro unidades (Fig. 3):

- Unidad 1: 0-2000 mm. Caracterizada por los mayores valores de los parámetros magnéticos $\left(\chi_{\mathrm{ARM}}, \mathrm{IRM}, \mathrm{HIRM}\right)$, especialmente en los primeros $500 \mathrm{~mm}$. Esta 
unidad presenta valores medios de TOC (0.6-0.7\%) y de elementos geoquímicos asociados a los aportes detríticos.

- Unidad 2: 2000-2400 mm. Esta unidad viene determinada por el claro descenso en el TOC (valores $<0.5 \%$ ) además de continuar el descenso en los parámetros magnéticos.

- Unidad 3: 2400-4500 mm. Es en esta unidad donde aparecen los valores más altos de TOC de toda la secuencia (1\%) y, además, se observan picos en los valores de $\mathrm{Ca}$ y de $\mathrm{Sr}$ asociados a láminas de carbonatos. Varias de estas láminas se caracterizan también por un alto valor de Mn. En la unidad 3 disminuyen algunos de los parámetros magnéticos $\left(\chi_{\mathrm{ARM}}\right)$ pero sube el S-ratio y aumenta la variabilidad en los parámetros L y $\chi_{\mathrm{ARM}} / \mathrm{SIRM}$.

- Unidad 4: 4500-5780 mm. El TOC decrece a lo largo de esta unidad hasta valores por debajo de $0.5 \%$ pero se observa un ligero aumento en el TIC (acompañado de $\mathrm{Ca}$ y $\mathrm{Sr}$ ). Las propiedades magnéticas $\chi_{\mathrm{ARM}} \mathrm{y}$ el S-ratio disminuyen mientras que hay un ligero aumento en la cantidad de $\mathrm{Ti}, \mathrm{K}, \mathrm{Si}, \mathrm{Rb}$, marcando un aumento en el contenido en partículas siliciclásticas.

\subsection{Marco cronológico}

El modelo de edad se ha obtenido a partir de las dataciones de ${ }^{14} \mathrm{C}$ presentadas en la tabla 2, descartando las muestras 1, 5 y 13 cuyos valores son incoherentes con su posición estratigráfica. Además, para el modelo de edad se ha considerado que el techo de la secuencia corresponde a la sedimentación actual (AD 2011), corroborado tanto por la conservación de la interfase sedimento-agua en los sondeos cortos como por la presencia de ${ }^{137} \mathrm{Cs}$ en los sedimentos superiores. La tasa de sedimentación media reciente $(0.86$ $\mathrm{mm} / \mathrm{año}$ ) calculada en el sondeo corto MAR11-1G-1A-4 mediante ${ }^{210} \mathrm{~Pb}$ y ${ }^{137} \mathrm{Cs}$ es mucho mayor que las tasas de sedimentación definidas con ${ }^{14} \mathrm{C}: 0.05 \mathrm{~mm} / \mathrm{año}$ en el sondeo corto MAR11-1G-1A-1 y 0.12 mm/año en el sondeo largo MAR11-1U-1A-1, lo que sugiere la existencia de un envejecimiento general de las dataciones con ${ }^{14} \mathrm{C}$. Este problema, denominado efecto reservorio, es común cuando se data materia orgánica de origen lacustre en lagos en los que una parte considerable del carbono utilizado por los organismos lacustres procede de la disolución de formaciones carbonatadas antiguas (Geyh et al., 1999). Dada la naturaleza carbonatada de la cuenca de recepción de Marboré un efecto reservorio era esperable.

Para calcular este envejecimiento, se ha obtenido con la regresión lineal que se aproxima a la datación de las muestras 2, 3 y 4 la edad que correspondería al techo de la secuencia. Así, el techo de la secuencia tendría una edad de 2265 años, lo que nos permite calcular un efecto reservorio, asumir que fue constante y restarlo a todas las dataciones por ${ }^{14} \mathrm{C}$. Esta corrección, junto con el hecho de que la tasa de sedimentación es muy homogénea en la secuencia de Marboré, permite obtener el marco cronológico de toda la secuencia mediante una regresión lineal (Fig. 5). De acuerdo con este modelo de edad, la secuencia de Marboré contiene los últimos 12.7 ka. 
EDAD cal B.P.

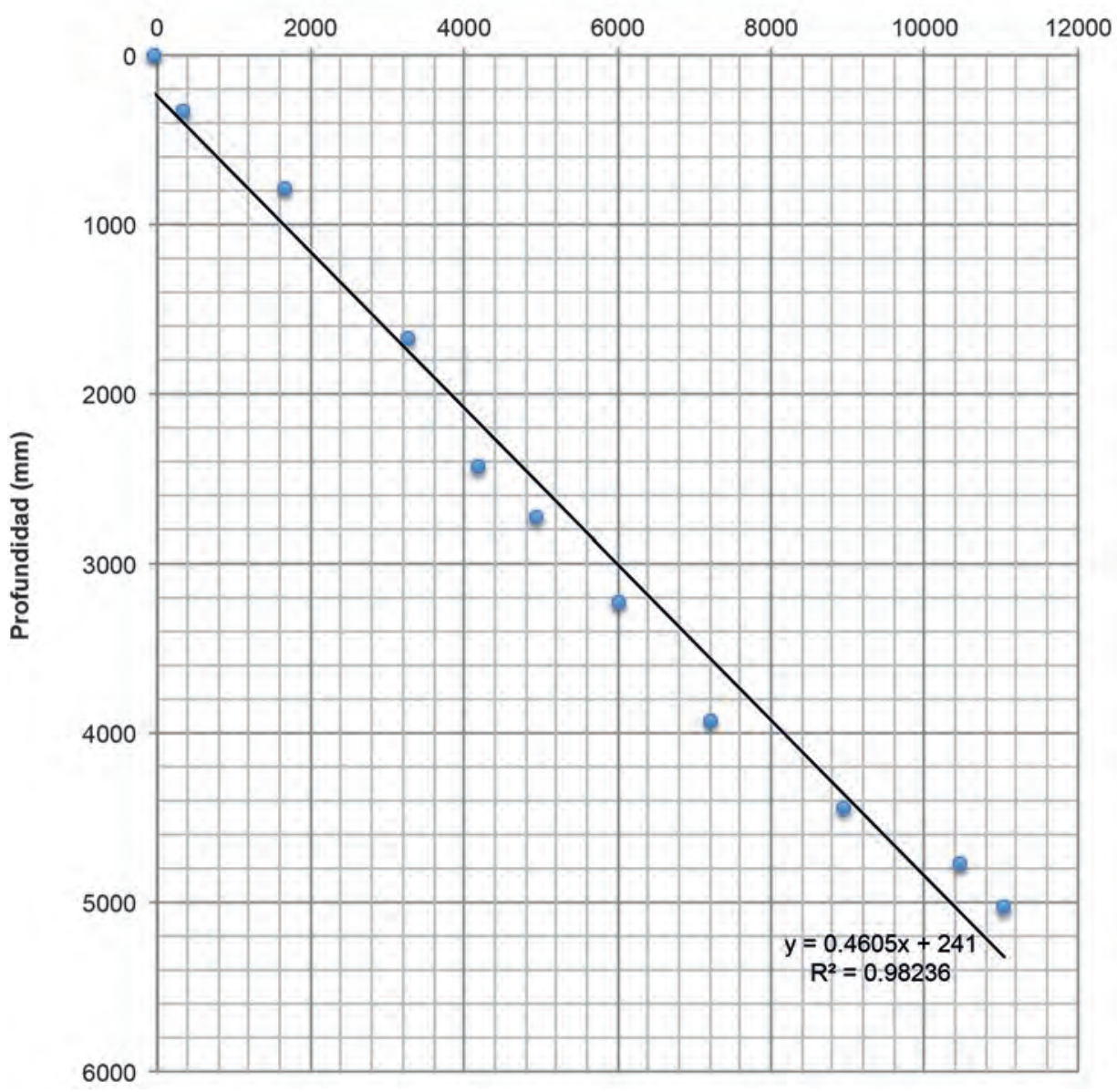

Figura 5. Modelo de edad corregido por el valor del efecto reservorio (ver texto para mayor explicación).

\section{Discusión}

El estudio multiproxy de la secuencia de Marboré permite analizar la evolución paleoclimática de esta región de montaña desde el inicio del Younger Dryas (12.7 ka) hasta nuestros días, y evaluar con especial énfasis las variaciones del contenido en minerales ferromagnéticos s.l. y su relación con los cambios ambientales.

\section{1. ¿Qué información ambiental proporcionan las propiedades magnéticas en el Lago de Marboré?}

Las variaciones de las propiedades magnéticas en sedimentos lacustres a lo largo del tiempo pueden responder a varios factores: i) cambio en el área de erosión, ii) cambio en 
la cantidad del aporte y por consiguiente en la erosión, iii) neoformación inducida por bacterias, y/o iv) variaciones diagenéticas con la consiguiente disolución/precipitación de minerales ferromagnéticos s.l.

El primero de estos factores puede ser descartado en la secuencia de Marboré por la alta correlación entre el Si y el Ti $(\mathrm{R}=0.66)$. La similitud entre estos dos elementos indica que el área de erosión no ha cambiado a lo largo del sondeo, ya que las variaciones relativas de concentración de elementos que no son favorables para la alteración diagenética (como son el Si y Ti) serían significativas si hubiese ocurrido un cambio en el área fuente (Ao et al., 2010). Por otro lado, la disminución o aumento en la cantidad de aporte al lago de Marboré tampoco parece ser la explicación de las variaciones magnéticas, ya que no se observa una variación del aporte detrítico en los análisis geoquímicos que hubiera llevado a grandes cambios en la tasa de sedimentación. De hecho, la variación en la erosión sugerida por las variaciones de elementos como Al, Si, K o Ti, no se correlaciona con ninguno de los parámetros magnéticos investigados. Este hecho podría indicar que la concentración de los minerales magnéticos y su variación a lo largo del sondeo no responden a un patrón local sobre la erosión en el entorno más próximo, sino que está asociada a factores más globales. La presencia de magnetita generada por la acción bacteriana es otro factor que puede ser descartado en la secuencia de Marboré. Así, el ratio $\chi_{\mathrm{ARM}} / \mathrm{SIRM}$,que informa sobre la presencia de magnetosomas bacterianos en el sedimento, tiene valores en el lago de Marboré siempre inferiores a $2 \times 10^{-3}$ $\mathrm{m} / \mathrm{A}\left(2 \mathrm{~cm}^{3} / \mathrm{emu}\right)$, lo que indica que no hay magnetita generada por bacterias (Moskowitz, 1993; Oldfield, 1999; Foster et al., 2008; Wang et al., 2010). Por último, la disolución diagenética de minerales ferromagnéticos s.l., que se ha descrito en lagos y en ambientes marinos (Oldfield, 1999), no parece tener una gran influencia en las propiedades magnéticas de los sedimentos de Marboré. Dicha disolución queda marcada por una disminución en los parámetros de concentración, y en la disminución del tamaño de grano, lo que representaría una disolución selectiva preferente de granos pequeños primero, por su mayor ratio superficie/volumen. Los parámetros que informan conjuntamente sobre la disolución de minerales ferrimagnéticos, serían las bajas concentraciones de $\chi_{\text {ARM }}$ /SIRM y valores altos de $S$-ratio (Snowball, 1993; Dearing, 1999) que no se observa en el lago de Marboré. Otro parámetro sería la susceptibilidad de alto campo (indicativa de granos superparagnéticos) que no se ha medido en Marboré (Wang et al., 2010; Foster, 2008). Por último, el hecho de que los valores de tamaño de grano obtenidos a partir de los ciclos de histéresis no indican una pérdida específica de los tamaños de grano más pequeños y que no se observa una correlación específica entre las variaciones de $\chi_{\text {ARM }}$ /SIRM con las del $S$-ratio, permite descartar una disolución diagenética (Ao et al., 2010).

Por lo tanto, las variaciones en las propiedades magnéticas analizadas en la secuencia de Marboré y, muy especialmente, el cambio de tendencia ocurrido hace unos 40005000 años en la concentración de elementos magnéticos, no pueden ser explicadas por cambios de escala local (cambios en el área fuente o en las tasas de erosión, alteración diagenética o producción de magnetita por bacterias) sino que responden a cambios ambientales de escala regional o global. 


\subsection{Desde el Younger Dryas hasta el presente: ¿qué nos cuenta la secuencia de Marboré sobre los cambios ambientales?}

El conjunto de indicadores analizados permite establecer la evolución paleoambiental durante los últimos $\sim 13 \mathrm{ka}$. Aunque el sondeo no alcanzó la base de la secuencia, es probable que el lago se iniciara poco después de los $13 \mathrm{ka}$ en coherencia con la datación del techo del paleolago de La Larri y la edad obtenida en la base del sondeo MAR11-1U-1A. Así, se ha dividido el periodo de tiempo estudiado en cuatro intervalos (Fig. 6). La Unidad 4, la inferior, comprende desde el inicio del YD hasta hace aproximadamente 9200 años. Esta etapa está caracterizada por los bajos valores de materia orgánica, contenidos ligeramente más elevados que en el resto de la secuencia de los elementos detríticos y por los abundantes picos de Ca y Mn. Además, destaca la variabilidad de los parámetros magnéticos al comienzo del Holoceno, especialmente debido a un descenso brusco en los minerales de alta coercitividad (variaciones en el parámetro $\chi_{\mathrm{ARM}}$ /SIRM especialmente). Los picos de Ca son especialmente marcados en la base de la secuencia, coincidiendo con el YD, lo que indica que las condiciones climáticas de ese periodo favorecieron la llegada de mayor cantidad de partículas carbonatadas del área fuente de manera que no se disuelven completamente en el lago. Probablemente, tras la retirada del glaciar había mucho material erosionado disponible que fue arrastrado y sedimentado en el lago de Marboré dando lugar al ligero aumento en Ti, K, etc. y a los picos de $\mathrm{Ca}$. El entorno, completamente desnudo de vegetación y muy influido por la dinámica glaciar y periglaciar, no favorecía la acumulación de materia orgánica. Posteriormente, tras la entrada al Holoceno y hasta hace 9200 años, se produce un aumento progresivo de la materia orgánica y una disminución de los aportes terrígenos. Ambas características apuntan hacia una leve mejora climática en estas zonas elevadas. Se observan además otras variaciones en los parámetros magnéticos (ligero ascenso en la concentración de minerales de coercitividad baja, ver ARM).

El intervalo temporal con un clima más benigno en los últimos 13000 años fue el correspondiente a la Unidad 3, entre 9200 y 4150 cal BP. Durante este periodo es cuando hay una mayor concentración de TOC, y siguen apareciendo picos de Ca y una mayor frecuencia de picos de Mn. Estos picos corresponden a intervalos con las láminas de mayor tamaño de grano, lo cual sugiere mayor frecuencia de aportes detríticos (aumento del Ca) y de la oxigenación en el fondo del lago y, por tanto, depósito del Mn formando óxidos. Algunos de estos máximos son coincidentes con el Fe, sugiriendo periodos de gran variabilidad en el equilibrio redox, quizás por oscilaciones del nivel del lago o por variaciones en la cantidad y tipo de los aportes detríticos. Durante este periodo la concentración de los minerales de baja coercitividad (magnetita) es más alta (S-ratio). Es interesante observar en este periodo, al comparar los datos geoquímicos con los magnéticos, que aparecen picos de $\mathrm{Ca}$ (relacionados en ocasiones con picos de $\mathrm{Mn}$ y $\mathrm{Sr}$ ) a la vez que desaparecen los minerales ferromagnéticos de alta coercitividad magnética y disminuyen los minerales ferromagnéticos de coercitividad baja (S-ratio, $\chi_{\mathrm{ARM}} / \mathrm{SIRM}$ ) (Fig. 6). Es especialmente significativo el pico de Ca que ocurre a 5500 cal yr BP ya que concurre con un aumento en $\mathrm{Mn}, \mathrm{Sr}$ y Fe y un descenso en TOC y elementos indicadores del aporte detrítico (K, Ti). Este conjunto de indicadores señalan un cambio ambiental signi- 
EDAD (cal yr. B.P.)

\begin{tabular}{|r|c|c|c|c|}
\hline \multicolumn{2}{|c|}{ Pleistoceno } & \multicolumn{3}{|c|}{ Holoceno } \\
\hline & Y.D. & Temprano & Medio & Reciente \\
\hline
\end{tabular}

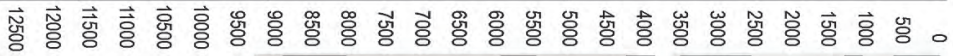

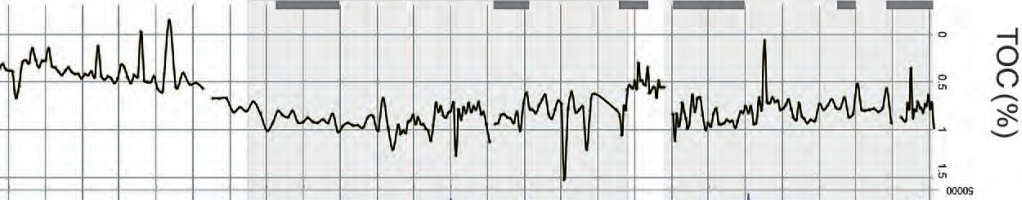

ô.

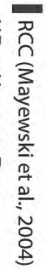

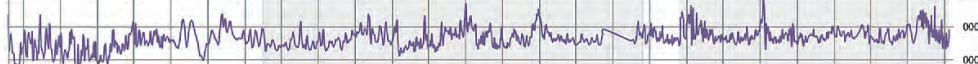

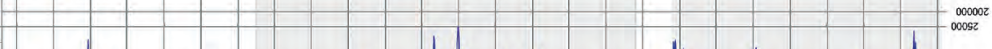

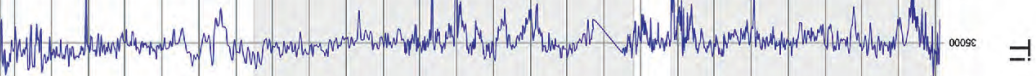

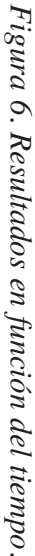

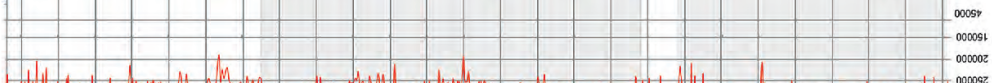

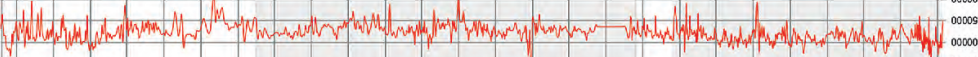

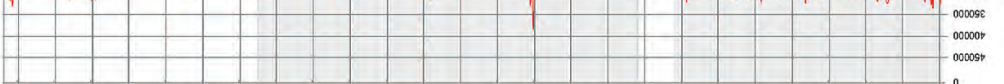

?

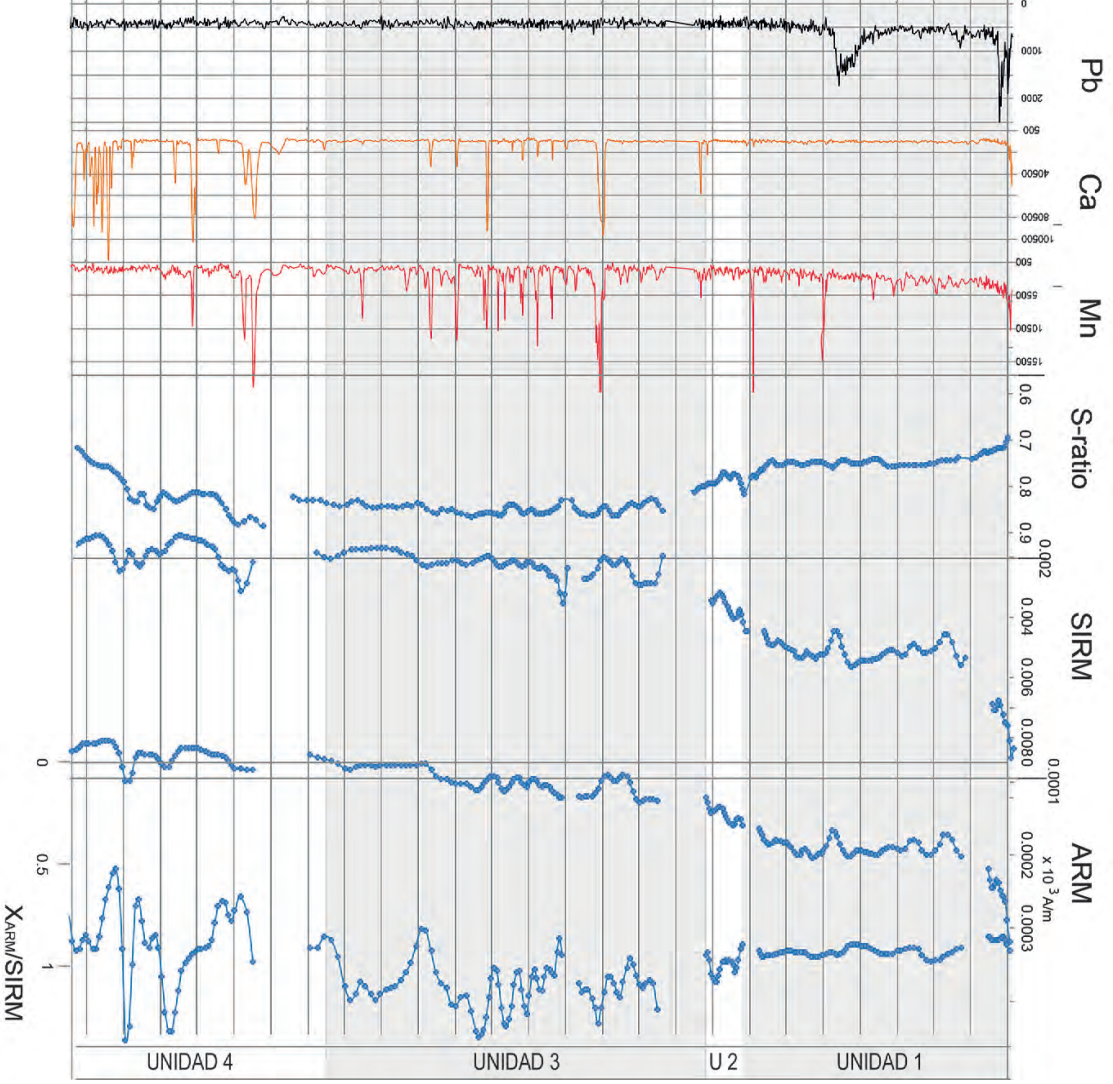


ficativo que ocurre en torno a los 5500 años cal. BP. Este cambio en las condiciones del lago afectó tanto a los elementos indicadores de cambios redox (Mn) como a los minerales ferromagnéticos s.l. (óxidos de Fe). Este periodo hace unos 5.5 ka corresponde a la transición entre el Holoceno temprano y el Holoceno medio y está relacionado con un aumento en la aridez (Jalut, et al., 2000; Pérez-Obiol et al., 2011). Además es sincrónico con un cambio climático abrupto (RCC por las siglas en inglés) de Mayewski et al. (2004) que se caracterizaría por condiciones frías y secas. En el Pirineo Montserrat-Martí (1992) y González-Sampériz et al. (2006), detectan en los lagos de Tramacastilla y Portalet el inicio de condiciones más secas a partir de 8-7.5 ka BP. En Marboré, situado a mayor altura, este cambio ocurriría dos milenios más tarde. Los datos magnéticos disponibles responden a una tendencia generalizada del paso de condiciones más húmedas a áridas, con una mayor acumulación (o menor destrucción) de estos minerales durante el periodo con mayor aridez. La presencia de minerales ferromagnéticos de alta coercitividad (hematites), que se da en la parte alta del sondeo de Marboré, se ha asociado (en lagos tropicales) a momentos de mayor aridez en condiciones óxicas (Williamson et al., 1998). No sólo comienza a haber mayor contenido de minerales de alta coercitividad, sino de baja, en la parte alta del sondeo. Este aumento en la concentración de los minerales ferromagnéticos s.l., no parece depender de la cantidad de carbono orgánico ni de la presencia de $\mathrm{S}$ (ambos en cantidades muy pequeñas), ya que la presencia de TOC y S puede provocar variaciones diagenéticas en los óxidos de hierro (ferromagnéticos s.l.). Sin embargo, medidas del Fe y $\mathrm{S}$ de muestras de la parte superior del sondeo (1235 $\mathrm{mm}$ ) en ICP-OES (CEBAS-CSIC, Murcia) permiten controlar que la presencia de S es muy baja en la secuencia (como el TIC y TOC) y que la correlación de su variación respecto al hierro es también muy baja $(\mathrm{R}=0.2)$. Además, los datos de fluorescencia de rayos $\mathrm{X}$ establecen la falta de correlación del Fe con los aportes detríticos de una forma unívoca, ya que el Fe no sólo depende de los aportes detríticos (en filosilicatos) sino de las condiciones redox (como el Mn, Levy et al., 1992).

Tras el cambio principal de la secuencia en torno a los 5500 años, tiene lugar un periodo de transición hasta aproximadamente 3650 cal BP que se caracteriza por una disminución en el TOC y un aumento en los parámetros que indican concentración de minerales ferromagnéticos s.l. A partir de ahí, el aumento en los parámetros magnéticos como el SIRM o ARM es ya muy significativo, al igual que la subida del TOC y la ausencia de picos de Ca. Este último intervalo entre hace 3650 años y el presente se caracteriza por la presencia de dos picos muy claros de $\mathrm{Pb}$, que parecen estar relacionados con la actividad antrópica en la zona (Salabarnada, 2011).

\section{Conclusiones}

El sondeo MAR11-1U-1A de casi $6 \mathrm{~m}$ de longitud, debido a sus características sedimentológicas y a la linealidad deducida en la tasa de sedimentación, supone una acumulación muy constante a lo largo de los últimos 12.7 ka, de 0.45 mm/a. Los sedimentos son limos y lutitas de color marrón y ocre compuestas esencialmente de cuarzo, minerales de la arcilla y otros silicatos con escasos porcentajes de materia orgánica y prácticamente sin carbonatos. Las laminaciones milimétricas observadas a partir de 
cambios de color se deben al diferente tamaño de grano y composición (blanca menor tamaño y mayor contenido en cuarzo y marrón mayor tamaño de grano y mayor contenido de biotitas). Las láminas grises presentan un porcentaje similar a las blancas en cuanto a contenido mineral, pero el tamaño de grano es mayor.

Se han definido 4 unidades dependiendo de los marcadores (proxies) analizados. Así, se ha identificado el periodo del YD marcado por los mayores valores de carbonatos de la secuencia, probablemente asociado a condiciones frías y mucha erosión. Posteriormente, tiene lugar un periodo más húmedo en el Holoceno temprano-medio que se corresponde con la Unidad 3, caracterizado por un pequeño aumento en el contenido en TOC, presencia de carbonatos detríticos, y una disminución generalizada en la concentración y variedad de minerales ferromagnéticos s.l. El cambio más importante tiene lugar al final de esta Unidad 3 (2400 mm, 4156 cal yr BP) y viene acompañado del aumento en la concentración de minerales ferromagnéticos s.l. (de baja y alta coercitividad). Esta variación se ha interpretado como un cambio a condiciones de mayor aridez.

\section{Agradecimientos}

Proyecto financiado por el Organismo Autónomo de Parques Nacionales HORDA: Dinámica glacial, clima y vegetación en el Parque Nacional de Ordesa-Monte Perdido durante el Holoceno (ref: 83/2009).

\section{Referencias bibliográficas}

Ao, H., Deng, CH., Dekkers, M.J., Liu, Q. 2010. Magnetic mineral dissolution in Pleistocene fluviolacustrine sediments, NihewanBasin (North China). Earth and Planetary Science Letters 292 191-200.

Arenas, C., Millán, H., Pardo, G., Pocoví A. 2001. Ebro Basin continental sedimentation associated with late compressional Pyrenean tectonics (north-eastern Iberia); controls on basin margin fans and fluvial systems. Basin Research 13 (1), 65-89.

Borruel Abadía, V. 2012. Magnetismo ambiental, indicador de la variabilidad climática en el entorno del Lago de Sanabria (últimos 26000 años). Trabajo Fin de Máster. Universidad de Zaragoza, 63 pp.

Cámara, P., Klimowitz J. 1985. Interpretación geodinámica de la vertiente centro-occidental surpirenaica (cuencas de Jaca-Tremp). Estudios Geológicos 41, 391-404.

Cohen, A.S. 2003. Paleolimnology. The history and evolution of lake systems. Oxford University Press, New York, 528 pp.

Channel, J.E.T., McCabe, C. 1994. Comparison of magnetic hysteresis parameters of unremagnetized and remagnetized limestones. Journal of Geophysical Research 99, 4613-4623.

Chueca, J., Julián, A., Peña-Monné, J.L. 2002. Comparación de la situación de los glaciares del Pirineo español entre el final de la Pequeña Edad del Hielo y la actualidad. Boletín Glaciológico Aragonés 3, 13-41.

Day, R., Fuller, M., Schmidt, V.A. 1977. Hysteresis properties of titanomagnetites: grain size and composition dependence. Physics of the Earth and Planetary Interiors 13, 260-267.

Dearing, J.A. 1999. Holocene Environmental Change from Magnetic Proxies in Lake Sediments. En Quaternary Climates, Environments and Magnetism, B.A. Maher, R. Thompson (eds.), Cambridge University Press, Cambridge, pp. 231-278. 
Dunlop, D.J. 2002. Theory and application of the Day plot $\left(\mathrm{M}_{\mathrm{rs}} / \mathrm{M}_{\mathrm{s}}\right.$ versus $\left.\mathrm{H}_{\mathrm{cr}} / \mathrm{H}_{\mathrm{c}}\right)$ 1. Theoretical curves and tests using titanomagnetite data. Journal of Geophysical Research 107 (B3). Doi: 10.1029/2001JB000486.

Foster, I.D.L., Oldfield, F., Flower, R.J., Keatings, K. 2008. Mineral magnetic signatures in a long core from Lake Qarun, Middle Egypt. Journal of Paleolimnology 40 (3), 835-849.

García-Ruiz, J.M., Moreno, A., González-Sampériz, P., Valero-Garcés, B., Martí-Bono, C. 2010. La cronología del último ciclo glaciar en las montañas del sur de Europa. Una revisión. Cuaternario y Geomorfología 24, 35-46.

García-Ruiz, J.M., Martí-Bono, C., Valero, B., González-Sampériz, P. 2001. La evolución de los glaciares del Pleistoceno Superior en el Pirineo Central español. El ejemplo de los glaciares de Escarra y Lana Mayor, Alto Valle del Gállego. Cuaternario y Geomorfología 15, 103-120.

García-Ruiz, J.M., Valero-Garcés, B.L., Martí-Bono, C., González-Sampériz, P. 2003. Asynchroneity of maximum glacier advances in the central Spanish Pyrenees. Journal of Quaternary Science 18, 61-72.

Geyh, M.A., Grosjean, M., Núñez, L., Schotterer, U. 1999. Radiocarbon Reservoir Effect and the Timing of the Late-Glacial/Early Holocene Humid Phase in the Atacama Desert (Northern Chile). Quaternary Research 52, 143-153.

González-Sampériz, P., Valero-Garcés, B.L., Moreno, A., Jalut, G., García-Ruiz, J.M., MartíBono, C., Delgado-Huertas, A., Navas, A., Otto, T., Dedoubat, J.J. 2006. Climate variability in the Spanish Pyrenees during the last 30,000 yr revealed by the El Portalet sequence. Quaternary Research 66, 38-52.

Huckriede, H., Meischner, D. 1996. Origin and environment of manganese-rich sediments within black-shale basins. Geochimica et Cosmochimica Acta 60 (8), 1399-1413.

Hunt, C.P., Moskowitz, B.M., Banerjee, S.K. 1995. Magnetic properties of rocks and minerals. En Rock Physics \& Phase Relations: A Handbook of Physical Constants, AGU Ref. Shelf, vol. 3, T.J. Ahrens, (ed.), AGU, Washington, D.C., pp. 189-204. Doi: 10.1029/RF003p0189.

Jackson, M. 1990. Diagenetic sources of stable remanence in remagnetized Paleozoic cratonic carbonates: A rock magnetic study. Journal of Geophysical Research 95, 2753-2761.

Jalut, G., Amat, A.E., Bonnet, L., Gauquelin, T., Fontugne, M. 2000. Holocene climatic changes in the Western Mediterranean, from south-east France to south-east Spain. Palaeogeography, Palaeoclimatology, Palaeoecology 160, 255-290.

Johnsen, S., Clausen, H., Dansgaard, W., Fuhrer, K., Gundestrup, N., Hammer, C., Iversen, P., Jouzel, J., Stauffer B., Steffensen, J. 1992. Irregular glacial inter-stadials recorded in a new Greenland ice core. Nature 359, 311-313.

King, J.W., Channel, J.E.T. 1991. Sedimentary magnetism, environmental magnetism, and magnetostratigraphy. Reviews of Geophysic Suppl. (IUGG Report-Contributions in Geomagnetism and Paleomagnetism) 29, 358-370.

Kodama, K.P., Lyons, J.C., Siver, P.A., Lott, A.M. 1997. A mineral magnetic and scaled-chrysophyte paleolimnological study of two northeastern Pennsylvania lakes: records of fly-ash deposition, land-use change, and paleorainfall variation. Journal of Paleolimnology 17, 173-189.

Labaume, P., Séguret, M., Seyve, C. 1985. Evolution of a turbiditic foreland basin an analogy with an accretionary prism: Example of the Eocene South-Pyrenean basin. Tectonics 4, 661-685.

Lanci, L., Lowrie, W., Wezel, F.C. 1995. Magnetic properties of Pleistocene sediments from the Mediterranean sea: a correlation with the paleoclimatic record. Terra Nova 7, 535-543.

Larrasoaña, J.C., Ortuño, M., Birks, H.H., Valero-Garcés, B., Parés, J.M., Copons R., Camarero, L.L., Bordonau, J. 2010. Palaeoenvironmental and palaeo-seismic implications of a 3700year sedimentary record from proglacial Lake Barrancs (Maladeta Massif, Central Pyrenees, Spain). Palaeogeography, Palaeoclimatology, Palaeoecology 294, 83-93. 
Levy, D.B., Barbarick, K.A., Siemer, E.G., Sommers, L.E. 1992. Distribution and partitioning of trace metals in contaminated soils near Leadville, Colorado. Journal of Environmental Quality 21, 185-195.

Leswis, C.J., McDonald, E.V., Sancho, C., Peña, J.L., Rhodes, E.J. 2009. Climatic implications of correlated Upper Pleistocene glacial and fluvial deposits on the Cinca and Gállego Rivers (NE Spain) based on OSL dating and soil stratigraphy. Global and Planetary Change 67, 141-152.

Liu, Q., Hu, P., Torrent, J., Barrón, V., Zhao, X., Jiang, Z., Su, Y. 2010. Environmental magnetic study of a Xeralf chronosequence in northwestern Spain: Indications for pedogenesis. Palaeogeography, Palaeoclimatology, Palaeoecology 293, 144-156.

Liu, X.M., Hesse, P., Rolph, T. 1999. Origin of maghaemite in Chinese loess deposites: aeolian or pedogenic? Physics of the Earth and Planetary Interiors 112, 191-201.

Maher, B.A., Thompson, R. 1999. Quaternary Climates, Environments and Magnetism. Cambridge University Press, Cambridge, $391 \mathrm{pp}$.

Martínez de Pisón, E., Arenillas, M. 1988. Los glaciares actuales del Pirineo español. En La nieve en el Pirineo Español, MOPU, Madrid, pp. 29-98.

Mayewski, P.A., Rohling, E.J., Stager, J.C., Karlèn, W., Maasch, K.A., Meeker, L.D., Meyerson, E.A., Gasse, F., Van Kreveld, S.A., Holmgren, C.A., Lee-Thorp, J.A., Rosqvist, G., Rack F., Staubwasser, M., Schneider, R., Steig, E.J. 2004. Holocene climate variability. QuaternaryResearch 62, 243-255.

Montes, S. 1992. Sistemas deposicionales en el Eoceno medio-Oligoceno del Sinclinorio de Guarga (cuenca de Jaca, Pirineo central). En II Congreso Geológico de España, Tomo 2, Universidad de Salamanca, Salamanca, pp. 150-160.

Montserrat-Martí, J. 1992. Evolución glaciar y postglaciar del clima y la vegetación en la vertiente sur del Pirineo: estudio palinológico. Instituto Pirenaico de Ecología, Zaragoza, 147 pp.

Morellón, M., Valero-Garcés, B., Vegas-Villarrúbia, T., González-Sampériz, P., Romero, Ó., Delgado-Huertas, A., Mata, P., Moreno, A., Rico, M., Corella, J.P. 2009. Lateglacial and Holocene palaeohydrology in the western Mediterranean region: The Lake Estanya record (NE Spain). Quaternary Science Reviews 28, 2582-2599.

Moreno, A., López-Merino, A., Leira, M., Marco-Barba, J., González-Sampériz, P., Valero-Garcés, B., López-Sáez, J., Santos, L., Mata, P., Ito, E. 2011. Revealing the last 13,500 years of environmental history from the multiproxy record of a mountain lake (Lago Enol, northern Iberian Peninsula). Journal of Paleolimnology 46, 327-349.

Moskowitz, B.M., Frankel, R.B., Bazylinski, D.A. 1993. Rock magnetic criteria for the detection of biogenic magnetite. Earth and Planetary Science Letters 120, 283-300.

Muñoz, J.A. 1992. Evolution of a continental collision belt: ECORS-Pyrenees crustal balanced section. En Thrust tectonics, K. R. McClay (ed.), Chapman \& Hall, London, pp. 235-246.

Myrbo, A., Morrison, A., McEwan, R. 2011. Tool for Microscopic Identification (TMI). Disponible en: http://tmi.laccore.umn.edu.

Nicolás-Martínez, P.M. 1981. Morfología del circo de Tucarroya (Macizo Monte Perdido, Pirineo Aragonés). Cuadernos de Investigación Geográfica 7, 51-80.

Oldfield, F. 1991. Environmental magnetism. A personal perspective. Quaternary Science Reviews $10,73-85$.

Oldfield, F. 1999. Environmental magnetism: the range of applications. En Environmental Magnetism: A Practical Guide Technical Guide No. 6, J. Walden, F. Oldfield, J. Smith (eds.), Quaternary Research Association, London, pp. 212-222.

Oliva-Urcia, B. 2004. Geometría y Cinemática rotacional en las Sierras Interiores y Zona Axial (sector de Bielsa) a partir del análisis estructural y paleomagnético. Tesis Doctoral. Universidad de Zaragoza, Zaragoza, 290 pp. 
Opdyke, N.D., Channel, J.E.T. 1996. Magnetic stratigraphy. Academic Press, San Diego, 346 pp.

Ortega, B., Caballero, M., Lozano, S., Vilaclara, G., Rodríguez, A., 2006. Rock magnetic and geochemical proxies for iron mineral diagenesis in a tropical lake: Lago Verde, Los Tuxtlas. East-Central Mexico. Earth and Planetary Science Letters 250, 444-458.

Parish, M. 1984. A structural interpretation of a section of the Gavarnie nappe and its implications for Pyrenean geology. Journal of Structural Geology 6, 247-255.

Pérez-Sanz, A., González-Sampériz, P., Valero-Garcés, B.L., Moreno, A., Morellón, M., Sancho, C., Belmonte, A., Gil-Romera, G., Sevilla, M., Navas, A. 2011. Clima y actividades humanas en la dinámica de la vegetación durante los últimos 2000 años en el Pirineo Central: el registro palinológico de la Basa de la Mora (Macizo de Cotiella). Zubía 23, 17-38.

Pérez-Obiol, R., Jalut, G., Julià, R., Pèlachs, A., Iriarte, M.J., Otto, T., Hernández-Beloqui, B. 2011. Mid-Holocene vegetation and climatic history of the Iberian Peninsula. The Holocene 21, 75-93.

Peters, C., Dekkers, M.J. 2003. Selected room temperature magnetic parameters as a function of mineralogy, concentration and grain size. Physics and Chemistry of the Earth 28, 659-667. Doi: 10.1016/S1474-7065(03)00120-7.

Plà, S., Catalán, J. 2005. Chrysophyte cysts from lake sediments reveal the submillennial winter/ spring climate variability in the northwestern Mediterranean region throughout the Holocene. Climate Dynamics 24, 263-278.

Pocoví, A., Millán, H., Navarro, J.J., Martínez, M.B. 1990. Rasgos estructurales de la Sierra de Salinas y zona de los Mallos (Sierras Exteriores, Prepirineo, provincias de Huesca y Zaragoza). Geogaceta 8, 36-39.

Reimer, P.J., Baillie, M.G.L., Bard, E., Bayliss, A., Beck, J.W., Blackwell, P.G., Bronk Ramsey, C., Buck, C.E., Burr, G.S., Edwards, R.L., Friedrich, M., Grootes, P.M., Guilderson, T.P., Hajdas, I., Heaton, T.J., Hogg, A.G., Hughen, K.A., Kaiser, K.F., Kromer, B., McCormac, F.G., Manning, S.W., Reimer, R.W., Richards, D.A., Southon, J.R., Talamo, S., Turney, C.S.M., Van der Plicht, J., Weyhenmeyer, C.E. 2009. IntCal09 and Marine09 radiocarbon age calibration curves, 0-50,000 years cal BP. Radiocarbon 51, 1111-1150.

Ríos, L.M., Galera, J.M., Barettino, D. 1987. Mapa geológico de España, 1:50.000. Segunda serie. Hoja 30-8, Bujaruelo. ITGE, Madrid.

Roberts, A., Cui, Y., Verosub, L. 1995. Wasp-waisted hystersis loops: Mineral magnetic characteristics and discrimination of components in mixed magnetic systems. Journal of Geophysical Research 100, 17909-17924.

Salazar, A., Mata, P., Valero-Garcés, B.L. 2011. Late glacial lacustrine sediments in La-Larri (Pineta valley, Pyrenees, N. Spain). IAS abstract, pp. 28760-28760.

Salabarnada, A. 2011. Recent depositional evolution of a high altitude, alpine lake: Marboré lake (Ordesa-Monte Perdido National Park, Spanish Pyrenees). Trabajo Fin de Máster en Cambio Global (CSIC-UIMP), 46 pp.

Schnurrenbergen, D., Russell, J., Kelts, K. 2003. Classification of lacustrine sediments based on sedimentary components. Journal of Paleolimnology 29, 141-154.

Smol, J.P., Birks, H.J., Last, W. 2001. Tracking environmental change using lake sediments. Springer, Dordrecht, $548 \mathrm{pp}$.

Snowball, I.F. 1993. Geochemical control of magnetite dissolution in subarctic lake sediments and the implications for environmental magnetism. Journal of Quaternary Science 8, 339-346.

Soler, M., Puigdefábregas, C. 1970. Líneas generales de la geología del Alto Aragón occidental. Pirineos 96, 5-19.

Sugiura, N. 1979. ARM, TRM and magnetic interactions: Concentration dependence. Earth and Planetary Science Letters 42, 451-455. 
Tauxe, L., Mullender, T.A.T., Pick, A. 1996. Potbellies, wasp-waists, and superparamagnetism in magnetic hysteresis. Journal of Geophysical Research 101, 571-583.

Teixell, A. 1992. Estructura alpina en la transversal de la terminación occidental de la Zona Axial pirenaica. Tesis doctoral. Universitat de Barcelona, Barcelona, $252 \mathrm{pp}$.

Teixell, A. 1996. The Ansó transect of the southern Pyrenees: basement and cover thrust geometries. Journal of Geophysical Research 102, 20325-20342.

Thompson, R.A., Oldfield, F. 1986. Environmental magnetism. Allen \& Unwin, London, 225 pp.

Verosub, K.L., Roberts, A.P. 1995. Environmental magnetism: Past, present, and future. Journal of Geophysical Research 100, 2175-2192.

Wang, H., Liu, H., Zhu, J., Yin, Y. 2010. Holocene environmental changes as recorded by mineral magnetism of sediments from Anguli-nuur Lake, southeastern Inner Mongolia Plateau, China. Palaeogeography, Palaeoclimatology, Palaeoecology 285, 30-49.

Wang, H., Liu, H., Zhao, F., Yin, Y., Zhu, J., Snowball, I. 2012. Early- and mid-Holocene palaeoenvironments as revealed by mineral magnetic, geochemical and palynological data of sediments from Bai Nuur and Ulan Nuur, southeastern inner Mongolia Plateau, China. Quaternary International 250, 100-118.

Williamson, D., Jelinowska, A., Kissel, C., Tucholka, P., Gibert, E., Gasse, F., Massault, M., Taieb, M., Van Campo, E., Wieckowski, K. 1998. Mineral-magnetic proxies of erosion/ oxidation cycles in tropical maar-lake sediments (Lake Tritrivakely, Madagascar): paleoenvironmental implications. Earth and Planetary Science Letters 155, 205-219. 